\title{
基于大数据的图书馆业务管理研究
}

\section{Research on Library Business Management Based on Big Data}

\author{
黄建军 \\ Jianjun Huang
}

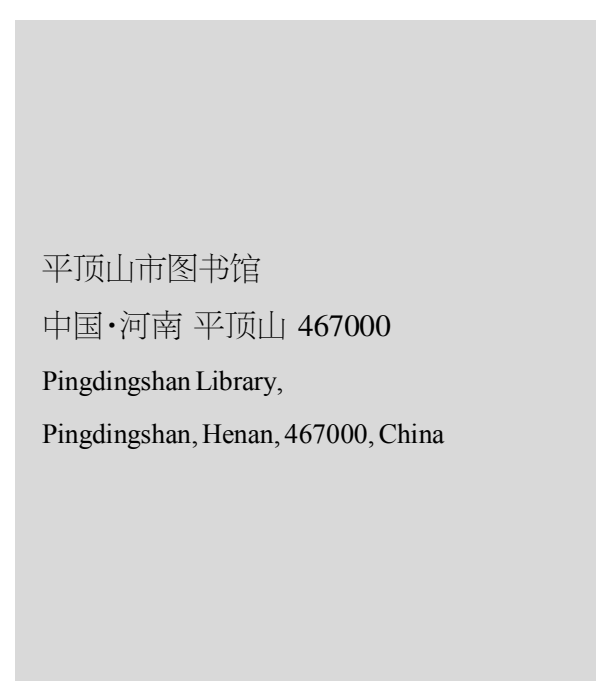

【摘要】在公共图书馆的发展过程中, 管理部门需要充分发挥大数据的作用, 注重大数 据分析服务, 将这项服务和社交网络进行融合, 强化图书馆业务管理力度, 培养图书馆工 作人员的数据分析能力, 进一步推动图书馆的发展。论文主要对大数据时代图书馆业务管 理的相关内容进行了分析。

【Abstract】In the process of the development of public libraries, the management departments need to give full play to the role of big data, note the major data analysis service, integrate this service with social networks, strengthen the management of library business, train the librarians' ability of data analysis, and further promote the development of libraries. This paper mainly analyzes the related contents of library business management in the big data era.

【关键词】大数据; 图书馆; 业务管理

【Keywords 】big data; library; operation control

【DOI】10.36012/lcs.v2i1.1458

\section{1 引言}

为了有效提升图书馆服务水平, 满足读者的阅读需求和 查阅需求, 图书馆必须利用大数据技术, 有效地进行调整和创 新,强化管理信息化、服务信息化建设,进一步提升图书馆业 务管理和服务水平, 为读者提供优质、高效的服务。

\section{2 大数据的相关内容}

在社会经济的发展和变革中, 众多新知识、新技术在各个 领域得到了有效应用, 大数据直接影响着人民群众的日常生 活, 使得人民群众的生活、学习和工作发生了很大改变 ${ }^{[1]}$ 。大数 据不仅是海量信息数据,还有更深层次的意义,但相关学者未 针对大数据概念给出准确的定论, 其定性未得到大家的认可。

大数据技术的特点如下: 首先, 信息容量大。大数据在图 书馆业务管理中得到了有效应用, 传统的图书馆业务管理模 式只是大数据业务管理中的一部分, 读者可以利用大数据技 术获取图书相关信息, 图书馆管理人员也可以借助大数据发 布更多信息, 使得图书馆内容更加丰富。其次, 信息类型的多 样性。在图书馆业务管理过程中, 会生成很多数据, 这些数据 类型具有一定的多样性, 庞大的数据类型会重新组成图书馆 大数据集。最后,信息数据处理较快。在图书馆业务管理过程 中, 管理部门可以利用云计算技术快速地分析图书馆的信息、
数据,为读者、图书管理人员提供准确的数据和信息。

\section{3 大数据时代图书馆业务管理发展机遇}

\section{1 转变图书馆服务方式}

在传统的图书馆业务管理服务过程中，管理人员普遍处 于被动状态, 无法为读者提供优质服务, 无法满足现代化社会 发展的实际需求。为了有效改善这一现状, 图书馆必须注重大 数据技术的应用,根据相关信息合理地选择范围,并对其进行 积累、描述、扫描等, 在做好各项处理工作后为读者提供个性 化的服务,如定期向读者提供信息推送服务,根据读者搜索的 信息、感兴趣的内容做好信息推送工作。

\section{2 提升图书馆服务水平}

在社会经济的快速发展中, 图书馆建设必须进行合理调 整, 根据现代化社会的发展, 满足大众的个性化需求, 为图书 馆的持续、稳定发展提供支持。在新时期, 图书馆需要不断提 升自身的服务水平, 拓宽服务内容, 这样才能满足时代的发展 需求, 以此为基础探索出满足自身发展需求的道路, 而大数据 技术的应用可以实现这一目标。在图书馆建设过程中, 相关部 门利用大数据技术处理并应用各项数字资源, 提供更加优质 的服务, 提高图书馆服务的整体水平。

\section{3 有效整合图书馆资源}

在现代化社会的发展中, 中国数字图书馆建设仍处于封 
数字图书馆 Digital Library

闭状态, 很多数字资源无法实现共享, 这就会带来严重的重复 建设、资源浪费等问题，不利于图书馆业务管理工作的顺利实 施。为了有效改善这一现状, 图书馆需要引进大数据技术, 从 而有效地解决这些问题, 实现图书馆内部各项资源的共享, 为 各个图书馆资源共享、整合提供支持。

\section{4 大数据时代图书馆业务管理问题与挑战}

在大数据时代, 图书馆业务管理遇到了很多问题, 面临着 很大挑战, 具体体现在以下四个方面: 首先, 大数据技术在图 书馆业务管理中发挥着重要作用, 各项数据、信息在不断增 长, 读者的阅读需求呈现出多元化、个性化的特点, 因此图书 馆存储结构必须具备一定的合理性, 以满足读者的阅读需求。 其次, 大数据技术在图书馆业务管理模式中得到了有效应用, 但图书馆数据会被分散到各个服务、管理平台中,为数据管理 工作带来了很大的困难，管理人员往往无法在频繁的信息移 动过程中获取更多有价值的信息 ${ }^{[2]}$ 。并且, 图书馆管理人员的 管理理念还有待更新，无法利用大数据技术为图书馆数据服 务,很难获取更多准确的信息。再次,在图书馆业务管理过程 中,大数据技术得到了有效应用,使得图书馆信息、数据日益 增加,管理人员需要确保图书馆数据中心信息的高效性、安全 性, 以满足图书馆的发展需求。最后, 在大数据时代, 图书馆业 务管理数据增长到一定值后, 图书馆数据运用值会有所下降, 图书馆需要利用大数据技术的优势, 优化传统的业务管理模 式,实行共享、开放、合作的业务管理模式与服务模式,为图书 馆数据、信息的共享提供支持。

\section{5 大数据时代图书馆业务管理的优化措施}

\section{1 构建动态采购平台}

在大数据时代, 图书馆需要将信息资源作为导向, 构建动 态采购平台, 不断提升图书馆业务管理的整体质量。在图书馆 经营、管理和服务过程中，信息资源是其中的基础和重点内 容, 为图书馆的发展提供了基础支持。图书馆需要利用大数据 的优势, 针对服务对象、图书馆经费情况做好数据分析工作, 以此明确图书馆采购类型, 确保图书馆大数据库的有效性。同 时, 图书馆需要利用大数据技术, 构建动态采购平台, 收集各 个层次读者的阅读信息、出版社动态、供应商的供应情况,并 设置相应的权限，利用云计算技术建立完善的图书馆评价制 度, 获取、分析并提取更多有价值的图书馆信息。在动态采购 平台运行的过程中, 图书馆需要渗透供应商信息、出版社信 息、书名信息,为读者的阅读提供更多便利,这样读者可以在 平台中选出自己需要的书籍, 而图书馆根据读者的选书记录,
深入分析选购书籍的种类, 实现图书馆资源的充分利用。

\section{2 提供个性化的精准服务}

图书馆中涉及大量与读者相关的数据和信息, 如读者在 访问过程中的流量数、读者的下载汶览信息、读者检索电子资 源产生的数据库日志、读者借还书过程中产生的流通日志、读 者各项社交网络数据等,图书馆可以利用这些信息、数据分析 读者的整体特征。这些数据和信息中涉及大量隐性行为、显性 行为, 相关人员需要对其进行深入研究, 突出数据和信息的价 值。同时, 图书馆工作人员探索数据特征和模型, 可以发现读 者群体的行为规律、阅读兴趣, 以此建立与读者个人相关的知 识体系, 明确读者的阅读需求, 为读者提供精准服务, 如个性 化推荐服务、主动式推送服务。例如, 淘宝、亚马逊等网站的个 性化商品推荐, 都是根据用户的访问记录为用户推荐相关的 专题信息, 根据用户的个性行为信息建立个性化用户行为模 型, 并根据模型相关规则进行挖掘, 为用户提供个性化服务, 在发现新的商品数据时及时向用户推送。另外, 图书馆可以积 极借鉴实践经验, 将其应用到图书馆建设和发展中, 为读者提 供个性化的精准服务。

\section{3 提升图书馆管理人员的综合素养}

在图书馆业务管理过程中, 需要强化管理人员业务管理 的培训, 明确业务管理模式, 并制定完善的培训制度, 为管理 人员提供自我提升的平台，不断提高图书馆管理人员的职业 素养。例如, 在图书馆培训过程中, 需要充分利用大数据技术, 根据管理人员的实际情况构建培训方案, 充分发挥出大数据 技术在图书馆业务管理中的作用, 提高管理人员的思想认知, 为图书馆业务管理水平的提升提供支持。除此之外, 图书馆需 要引进优秀的专业管理人员, 构建高素质的业务管理服务队 伍, 并且管理人员需要树立自主学习意识, 在自我学习的过程 中掌握信息技术的应用方法,提高自身的业务能力,可以借助 微课、慕课等,获取更多的学习资源。

\section{6 结语}

综上所述, 在图书馆业务管理过程中, 需要引进大数据 技术, 借助大数据的优势和特点做好业务管理工作, 确保各 项业务管理工作的智能性、精准性, 为图书馆的持续发展提 供保障。

\section{参考文献}

[1]孙冰洋. 探究大数据对图书馆业务应用与服务创新驱动 $[\mathrm{J}]$.办 公室业务,2019(24): 190+192.

[2]佟良,康莉.信息化环境下我国图书馆业务管理模式变革研究 [J].中国教育技术装备,2019(19):66-68. 